\title{
CURRENT TRENDS IN DIABETIC RETINOPATHY
}

\author{
Abdul Ahad Kazi, Nazir A. Laghari and Shafi Muhammad Jatoi
}

Diabetes mellitus is on the rise. There is an apparent epidemic of diabetes amongst adult populations of disadvantaged communities, both in developing countries and also in the industrialized world. According to National Health Survey of Pakistan $^{1}$, the prevalence of diabetes among population aged $\geq 25$ year is $4.2 \%$, while Shera et $\mathrm{al}^{2,3}$ have estimated a prevalence of 9.1 to $13.7 \%$. These figures translate into 4-8 million diabetics in the country. For every patient who is known to have diabetes another has the disease unaware. ${ }^{4}$ It is a significant cause of multi-system disease including cardiovascular disease, renal failure and blindness. Even though the diagnosis of diabetes mellitus may be anticipated by its classical signs and symptoms; weight loss, fatigue, a history of polydipsia (excessive thirst) and polyuria (excessive urinating), the diagnosis should not rely only on the presence of glycosuria (sugar in the urine). The diagnosis must be confirmed by demonstrating the presence of fasting hyperglycemia (raised blood sugar) and following an oral glucose tolerance test. ${ }^{5}$

Twenty years after the onset of diabetes nearly all patients with type I diabetes (insulin-dependent) and more than $60 \%$ of those with type II diabetes (non insulin-dependent) will have retinopathy. ${ }^{6}$ Diabetic retinopathy is the leading cause of blindness amongst people of working age in industrialized countries. ${ }^{7}$ It is estimated that there are 20 million diabetics in the Middle East (WHO Eastern Mediterranean Region), of which 800,000 will require treatment for retinopathy, and 116,000 new patients will present for treatment each year. ${ }^{8}$ In Singapore, $8.6 \%$ of the population between 15 and 69 years is diabetic, that is, 165,000 diabetics and at least 16,500 of these will have retinopathy ${ }^{9}$. Pilot studies performed by $\mathrm{Khan}^{10}$ and Kayani etal. ${ }^{11}$ suggest the prevalence of diabetic retinopathy between $26.1 \%$ and $33.3 \%$ in Pakistani diabetics. According to the US Census Bureau, International Data Base 2004 the extrapolated incidence (the number of new cases diagnosed each year) of diabetic retinopathy in Pakistan is 38,043 .

Diabetic retinopathy is a leading cause of ocular morbidity. Thylefors etal. in their analysis of the WHO Global Data Bank on Blindness have reported that the survey data on diabetic retinopathy as a cause of blindness is too limited to reach an accurate figure. ${ }^{12}$ However, it does seem likely that blinding diabetic eye disease is now the fourth major cause of blindness worldwide after cataract, glaucoma and trachoma. Diabetic retinopathy can cause several types of vitreoretinal pathology, each a consequence of microvascular damage to the retina. The 3 main categories are nonproliferative diabetic retinopathy (NPDR), proliferative diabetic retinopathy (PDR), and diabetic macular edema (DME). DME can coexist with any stage or severity of NPDR or PDR.

Although, the 'ideal screening method does not exist' and many in developing countries will not have available sophisticated equipment for screening, all primary care workers should be aware that diabetes is a public health problem in their region and make sure that diabetic patients have regular, dilated view ophthalmoscopy. The recommendations for screening of diabetic retinopathy by the American Academy of Ophthalmology and the American Diabetic Association are that; a newly diagnosed Type I diabetic should get their initial retina examination within 5 years after diagnosis. Follow-up examinations should be annual thereafter unless, there is moderate to severe diabetic retinopathy in which case examinations have to be more frequent. Newly diagnosed Type II diabetics should receive their initial examination immediately. Follow-up examinations may be conducted similarly to Type I diabetics. Early treatment using laser photocoagulation has been shown to reduce blindness due to diabetic retinopathy by at least $60 \%$ and the figure will improve further as patients at risk are identified early: the earlier the better. ${ }^{6}$ Late diagnosis of diabetic retinopathy may require complex vitreo-retinal surgical procedures for treatment and often leave the patient with irreversible loss of vision.

In conclusion, recognition of the increasing problem of diabetes mellitus and its danger to sight, training in ophthalmoscopy (and other diagnostic methods, if possible), early identification of the stages of retinopathy in patients at risk and referral for expert opinion and treatment, will prevent blindness for many individuals in vulnerable communities around the world. Blindness from diabetic retinopathy is almost inevitable if left unmanaged. Screening protocols need to be established at primary, secondary and tertiary referral centers at the provincial and national levels. Early identification of 
diabetes and diabetic retinopathy may allow earlier intervention, therefore, preventing the sequele of severe blindness in an already burdened economy.

\section{REFERENCES}

1. Pakistan Medical Research Council. National Health Survey of Pakistan, Islamabad: Pakistan Medical Research Council; 1997:54.

2. Shera AS, Rafique G, Khwaja IA et al. Pakistan National Diabetes Survey: prevalence of glucose intolerance and associated factors in Baluchistan province. Diabetes Res Clin Pract 1999; 44: 4958.

3. Shera AS, Rafique G, Khwaja IA et al. Pakistan national diabetes survey: prevalence of glucose intolerance and associated factors in Shikarpur, Sindh Province. Diabet Med 1995;12:1116-21.

4. Diabetes 2000 Project Leaflet, "Elimination of preventable blindness from diabetes by the year 2000". American Academy of Ophthalmology, USA.

5. WHO Technical Report Series 844, Prevention of diabetes mellitus: report of a WHO study group.
Geneva: WHO, 1994.

6. American College of Physicians, American Diabetes Association and American Academy of Ophthalmology. Screening guidelines for diabetic retinopathy. Ophthalmology 1992; 99: 1626-8.

7. Kohner EN, Porta M. Screening for Diabetic Retinopathy in Europe: A Field Guide-Book. Copenhagen: WHO Regional Office for Europe, 1992.

8. Ahmed AA. Prevention of blindness due to diabetes in the Middle East (Editorial). Middle East J Ophthalmol 1994; 2: 68-9.

9. Yeo KT, Lim ASM, Ling SL et al. Mass screening for diabetic retinopathy in the prevention of blindness. Asia-Pacific J Ophthalmol 1995; 7: 2-10.

10. Khan AJ. Prevalence of diabetic retinopathy in Pakistan subjects. A pilot study. J Pak Med Assoc 1991;41:49-50.

11. Kayani H, Rehan N, Ullah N. Frequency of retinopathy among diabetics admitted in a teaching hospital of Lahore. J Ayub Med Coll Abbottabad. 2003,15(4):53-6.

AUTHOR AFFILIATION:

Dr. Abdul Ahad Kazi (Corresponding Author)

Visiting Professor, Department of Ophthalmology

Liaquat University of Medical and Health Sciences

(LUMHS), Jamshoro, Sindh - Pakistan

Prof. Nazir A. Laghari

Department of Ophthalmology, LUMHS, Jamshoro

Prof. Shafi Muhammad Jatoi

Department of Ophthalmology, LUMHS, Jamshoro 